\title{
Blockade of TGF- $\beta$ inhibits mammary tumor cell viability, migration, and metastases
}

\author{
Rebecca S. Muraoka, ${ }^{1,2}$ Nancy Dumont, ${ }^{1}$ Christoph A. Ritter, ${ }^{3}$ Teresa C. Dugger, ${ }^{3}$ \\ Dana M. Brantley, ${ }^{3}$ Jin Chen, ${ }^{2,3}$ Evangeline Easterly, ${ }^{1}$ L. Renee Roebuck, ${ }^{3}$ Sarah Ryan, ${ }^{4}$ \\ Philip J. Gotwals, ${ }^{4}$ Victor Koteliansky, ${ }^{4}$ and Carlos L. Arteaga ${ }^{1,2,3}$
}

\begin{abstract}
${ }^{1}$ Department of Cancer Biology, Vanderbilt University School of Medicine, Nashville, Tennessee, USA
${ }^{2}$ Vanderbilt-Ingram Cancer Center, Nashville, Tennessee, USA

${ }^{3}$ Department of Medicine, Vanderbilt University School of Medicine, Nashville, Tennessee, USA

${ }^{4}$ Biogen Inc., Cambridge, Massachusetts, USA

Address correspondence to: Carlos L. Arteaga, Division of Oncology, Vanderbilt University School of Medicine, 2220 Pierce Avenue, 777 Preston Research Building, Nashville, Tennessee 27232-6307, USA.

Phone: (615) 936-3524; Fax: (615) 936-1790; E-mail: carlos.arteaga@mcmail.vanderbilt.edu.
\end{abstract}

Received for publication February 8, 2002, and accepted in revised form May 1, 2002.

TGF- $\beta$ s are potent inhibitors of epithelial cell proliferation. However, in established carcinomas, autocrine/paracrine TGF- $\beta$ interactions can enhance tumor cell viability and progression. Thus, we studied the effect of a soluble Fc:TGF- $\beta$ type II receptor fusion protein (Fc:T $\beta$ RII) on transgenic and transplantable models of breast cancer metastases. Systemic administration of Fc:T $\beta R I I$ did not alter primary mammary tumor latency in MMTV-Polyomavirus middle $\mathrm{T}$ antigen transgenic mice. However, Fc:T $\beta$ RII increased apoptosis in primary tumors, while reducing tumor cell motility, intravasation, and lung metastases. These effects correlated with inhibition of Akt activity and FKHRL1 phosphorylation. Fc:T $\beta$ RII also inhibited metastases from transplanted 4T1 and EMT-6 mammary tumors in syngeneic $\mathrm{BALB} / \mathrm{c}$ mice. Tumor microvessel density in a mouse dorsal skin window chamber was unaffected by Fc:T $\beta$ RII. Therefore, blockade of TGF- $\beta$ signaling may reduce tumor cell viability and migratory potential and represents a testable therapeutic approach against metastatic carcinomas.

J. Clin. Invest. 109:1551-1559 (2002). doi:10.1172/JCI200215234.

\section{Introduction}

Tumor metastases are the result of a complex process that involves cellular migration, tumor vascularization, interactions with the microenvironment, intravasation into blood or lymphatic vessels, and cell survival at distant sites (1). TGF- $\beta$ is a multifunctional cytokine involved in several of these processes $(2,3)$. The role of TGF- $\beta$ in the biology of epithelial cells is complex. TGF- $\beta$ potently inhibits the proliferation of epithelial cells (2). Transgenic mice that overexpress active TGF- $\beta 1$ in mammary epithelium exhibit hypoplastic mammary glands that are resistant to oncogene- or carcinogen-induced mammary cancers (4-6). In a mouse skin model of chemical carcinogenesis, expression of TGF- $\beta 1$ in keratinocytes suppresses the formation of benign skin tumors. Once tumors develop, however, TGF- $\beta 1$ enhances tumor progression to a highly invasive spindle cell phenotype (7). Ha-Ras-induced mammary tumor cells secrete high levels of TGF- $\beta$ and display highly invasive characteristics in vitro and in vivo (8). Introduction of dominant negative TGF- $\beta$ type II receptors (TRRII) into these cells retards primary tumor and metastases formation and prevents epithelial-to-mesencymal transition (EMT) (9). It appears, then, that many epithelial tumors escape growth inhibition by TGF- $\beta$, and TGF- $\beta$ secretion by cancer and/or stromal cells may contribute to late tumor progression. Tumor TGF- $\beta$ secretion may also indirectly favor metastatic progression by increasing extracellular matrix production/degradation, inducing tumor vascularization, and inhibiting effector mechanisms of immune surveillance $(3,10)$.

We have investigated the effect of TGF- $\beta$ on breast cancer metastasis using a soluble chimeric protein composed of the extracellular domain of the T $\beta R I I$ and the $\mathrm{Fc}$ portion of the murine $\mathrm{IgG}_{1}$ heavy chain (Fc:T $\beta \mathrm{RII})$ (11). This chimera interferes with TGF- $\beta$ binding to endogenous TGF- $\beta$ receptors and has been shown to block TGF- $\beta$-induced fibrosis in vivo (12).

\section{Methods}

Fc:T $\beta R I I$ and transgenic mice. Fc:T $\beta R I I$ has been described previously (11). FVB MMTV-Polyomavirus middle T antigen (MMTV-PyV mT) mice (13) (The Jackson Laboratories, Bar Harbor, Maine, USA) were housed in the Animal Care Facility at Vanderbilt University following The American Association for the Accrediation of Laboratory Animal Care guidelines. Three-week-old transgenic mice were treated twice weekly with Fc:T $\beta R I I$ in PBS $(5 \mathrm{mg} / \mathrm{kg})$ by intraperitoneal injection. At 110 days, tissues were harvested and fixed in formalin or were snap-frozen. Serum levels of Fc:T $\beta R I I$ were measured by 
immunoblot analysis using an anti-mouse $\mathrm{IgG}_{2 \mathrm{~A}}-\mathrm{HRP}$ (Southern Biotechnology Associates, Birmingham, Alabama, USA) against an Fc:T $\beta$ RII standard curve (3.3-66 nM).

Histological analyses. Paraffin sections $(5 \mu \mathrm{m})$ were stained with hematoxylin and eosin (Sigma-Aldrich, St. Louis, Missouri, USA). For immunohistochemistry, sections were treated as described (14), using Ab's against CD31 (1:100; Santa Cruz Biotechnology Inc., Santa Cruz, California, USA) or PyV mT antigen (pAb 701 [see ref. 15]; 1:50; provided by Steven Dilworth, Imperial Cancer Research Fund, London, United Kingdom). Immunohistochemical detection of bromodeoxyuridine (BrdU) incorporation and apoptosis was performed as described (16). Immunocytochemistry for Smad2, FKHRL1, vimentin, or $\beta$-catenin used Smad2 (1:100; Santa Cruz Biotechnology Inc.), FKHRL1 (1:100, Upstate Biotechnology Inc., Lake Placid, New York USA), vimentin (1:100; Santa Cruz Biotechnology Inc.), or $\beta$-catenin Ab's (Signal Transduction Laboratories, Lexington, Kentucky, USA), and Cy3-conjugated goat anti-rabbit IgG (Jackson ImmunoResearch Laboratories Inc., West Grove, Pennsylvania, USA).

Primary mammary tumor cell isolation and motility/invasion assays. Tumors from 110-day-old mice were digested $\left(37^{\circ} \mathrm{C}\right.$, 4 hours) in $3 \mathrm{mg} / \mathrm{ml}$ collagenase A (SigmaAldrich), washed (PBS/10\% FBS), and plated in DMEM:F12 (50:50; Life Technologies Inc., Carlsbad, California, USA), $5 \mathrm{ng} / \mathrm{ml} \mathrm{EGF,} 5 \mathrm{ng} / \mathrm{ml}$ 17- $\beta$ estradiol, 5 $\mathrm{ng} / \mathrm{ml}$ progesterone, and $50 \mathrm{ng} / \mathrm{ml}$ insulin (all from Sigma-Aldrich). For wound closure assays, primary mammary tumor cells (PMTCs) were grown to confluence, treated with $80 \mathrm{pM}(2 \mathrm{ng} / \mathrm{ml})$ TGF- $\beta 1,20 \mathrm{nM}$ Fc:T $\beta R I I$, or both, and wounded with a sterile circular rubber eraser ( $1 \mathrm{~cm}$ diameter). Cells were photographed at $0,8,16$, 24 , and 48 hours after wounding. The area of the circle enclosed by cells was determined using BioQuant (R\&M Biometrics, Nashville, Tennessee, USA) software. Experiments were conducted with and without mitomycin C (1 $\mu \mathrm{g} / \mathrm{ml}$; Sigma-Aldrich). For invasion assays, PMTCs, 4T1, or EMT6 cells $\left(10^{4}\right.$ each) were seeded in the upper chamber of transwells fitted with Matrigel-coated $8-\mu \mathrm{M}$ poresize polycarbonate filters (Corning Life Sciences, Acton, Massachusetts, USA). Lower chambers contained 2.5\% serum with or without $20 \mathrm{nM} \mathrm{Fc:T} \beta$ RII. After 24 hours, cells were scraped from upper filter surfaces, and the cells on the lower surfaces were stained and counted.

Western blot analyses. Total protein $(20 \mu \mathrm{g})$ was harvested and Western blot analysis performed as described previously (17) using the following Ab's: Shc, p85, Src, VEGF, and CD31 (all from Santa Cruz Biotechnology Inc.); Akt and Ser ${ }^{473}$ P-Akt (Transduction Laboratories, Lexington, Kentucky, USA); FKHRL1 and phospho-FKHRL1 (Upstate Biotechnology Inc.). Densitometric analysis was performed using ImageQuant software (Molecular Dynamics ImageQuant, Sunnyvale, California, USA).

${ }^{125}$ I-TGF- $\beta 1$ labeling. PMTCs were affinity labeled with 100 pM ${ }^{125}$ I-TGF- $\beta 1$ (NEN Life Science Products Inc.,
Boston, Massachusetts, USA) as described (18). In some cases, cross-linked cell lysates were precipitated with $\mathrm{Ab}$ $(1 \mu \mathrm{g})$ against type I (V22) or type II (C16) TGF- $\beta$ receptors (both from Santa Cruz Biotechnology Inc.). All samples were fractionated using 3-12\% gradient SDSPAGE, followed by autoradiography.

TGF- $\beta$ reporter assays. PMTCs $\left(0.5 \times 10^{6}\right)$ were transfected with p3TP-Lux $(2 \mu \mathrm{g})$ as described (19). After 48 hours, cells were treated for 6 hours with 80 pM TGF- $\beta 1$, $20 \mathrm{nM}$ Fc:T $\beta$ RII, or both. Luciferase activity was determined using the Dual Luciferase Assay system (Promega Corp., Madison, Wisconsin, USA).

Measurement of secreted TGF- $\beta$. PMTCs $\left(2 \times 10^{6}\right)$ or primary mammary epithelial cells from wild-type mice were cultured for 24 hours in $3 \mathrm{ml}$ of serum-free PMTC media. Conditioned medium was collected, concentrated to $0.5 \mathrm{ml}$, and TGF- $\beta 1$ levels in media were determined using a TGF- $\beta 1$ ELISA (R\&D Systems Inc., Minneapolis, Minnesota, USA).

Intravasation assay. Circulating tumor cells in 110-dayold PyVmT mice were quantified as described in Wyckoff et al. (20). One milliliter of blood was collected by heart puncture and centrifuged $\left(1200 \mathrm{~g}, 4^{\circ} \mathrm{C}, 5 \mathrm{~min}-\right.$ utes). The serum/buffy-coat layers were plated in $1 \mathrm{ml}$ DMEM:F12 (50:50)/10\% FBS. After 24 hours, the plates were washed with PBS to remove erythrocytes and nonadherent cells, and fresh DMEM:F12/10\% FBS was replenished. After 7 days, colonies were stained with hematoxylin and counted.

Matrix metalloproteinase activity. Mouse tumors were harvested in $25 \mathrm{mM}$ Tris- $\mathrm{HCl}$, pH 7.8, $0.5 \mathrm{M} \mathrm{NaCl}, 1 \%$ NP-40, and EDTA-free protease inhibitor cocktail (Roche Diagnostics GmbH, Mannheim, Germany). Fifty micrograms of protein was analyzed for matrix metalloprotease-2/matrix metalloproteinase-9 (MMP-2/ MMP-9) combined activity using the MMP Activity Assay Kit (Chemicon International Inc., Temecula, California, USA). The relative MMP activity per sample was compared with the MMP activity in $0.2 \mu \mathrm{g}$ of recombinant MMP-2 (Chemicon International Inc.).

Transplants/metastases of $4 T 1$ and EMT6 tumor cells. 4T1 or EMT6 cells $\left(0.5 \times 10^{5}\right)$ were injected into number 4 mammary glands of $\mathrm{BALB} / \mathrm{c}$ virgin female mice. Mice were treated with Fc:T $\beta R I I$ as described above. After 10 days, primary tumors were resected. Lungs were harvested 8 weeks later, and surface metastases were counted.

Dorsal skin window assay. Window chambers were prepared in dorsal skin folds of female BALB/c mice as described (21). 4T1-green fluorescent protein (4T1-GFP) (22) cells (1,000 cells) were implanted in the chambers along with $1 \mathrm{~mm}^{3}$ slow-release hydron pellets impregnated with Fc:T $\beta$ RII $(1 \mu \mathrm{g})$, normal mouse IgG $(1 \mu \mathrm{g})$, or PBS. Growth of 4T1-GFP cells and vessels was observed within the window from days $0-15$ using fluorescence and bright-field microscopy. On day $15,100 \mu$ l of rhodamine-conjugated dextran $(1 \mathrm{mg} / \mathrm{ml})$ was injected intravenously to label functional vessels. Fluorescence was quantified using Scion Image software. 
a
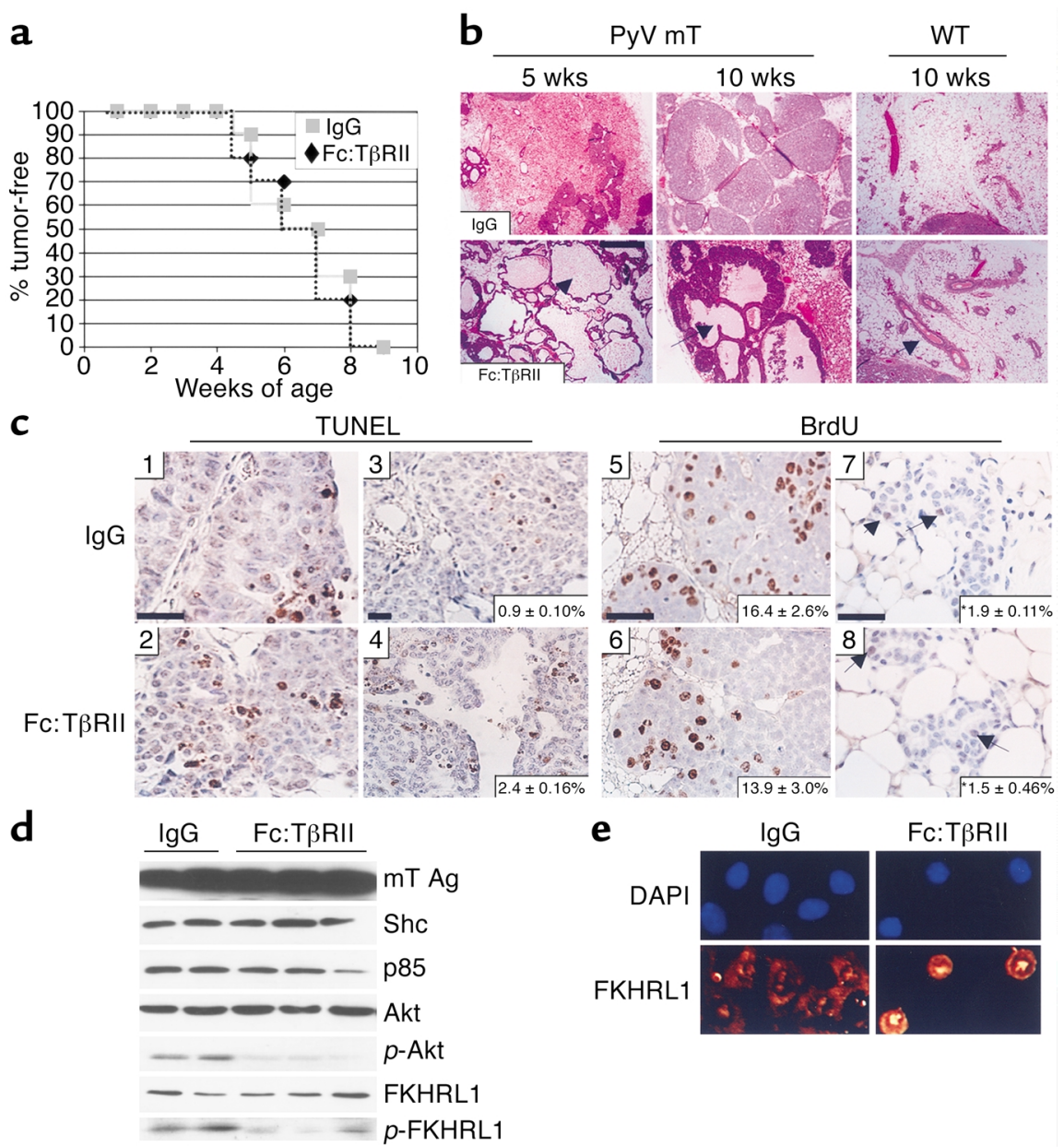

e

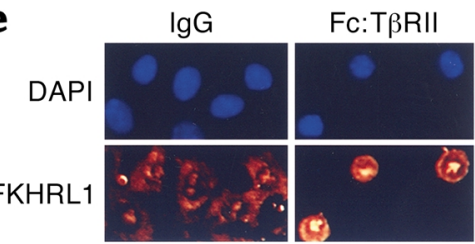

\section{Figure 1}

Tumor morphology, rate of apoptosis, and Akt signaling are altered by treatment with Fc:T $\beta R I I$. (a) MMTV/PyV $m T$ mice treated with Fc:T $\beta R I I$ or normal mouse IgG were examined twice weekly from 21 to 110 days of age. The initial observation of tumor onset is represented. (b) Histologic sections of tumors from 35- or 70-day-old transgenic or wild-type mice. Arrowheads indicate dilated ducts filled with secretory products. (c) TUNEL analysis (panels 1-4) of tumors harvested from 35- or 70-day-old mice treated with Fc:T $\beta$ RII or IgG. $n=6$ per condition. Quantification of percentage of apoptotic nuclei (bottom right corner of each panel) was calculated using the following equation: (number ofTUNEL-positive nuclei in $\times 400$ field) / (number of total nuclei in $\times 400$ field). BrdU incorporation analysis (panels 5-8) of tumors harvested from 70-day-old MMTV/PyV mT or wild-type mice. Arrowheads in panels 7 and 8 indicate BrdU-positive nuclei. Quantification of percentage of BrdU-positive nuclei (bottom right corner of each panel) was calculated using the following equation: (number of BrdU-positive nuclei in $\times 400$ field) / (number of total nuclei in $\times 400$ field). ${ }^{*} P=0.15$. Scale bars $=25 \mu \mathrm{m}$. (d) Tumor extracts harvested from 110 -day-old transgenic mice were subjected to Western blot analysis using Ab's against the mT Ag, Shc, p85, Akt, $p$-Akt, FKHRL1, and $p$-FKHRL. (e) PMTCs were incubated with or without $20 \mathrm{nM} \mathrm{Fc:T \beta RII} \mathrm{for} 6$ hours and stained with a FKHRL1 Ab followed by staining with Cy3-conjugated anti-rabbit Ab. Nuclei were counterstained with DAPI.

\section{Results}

Soluble Fc:TßRII fusion protein increases mammary tumor cell apoptosis in MMTV-PyV mT transgenic mice. Transgenic mice that express the PyV mT antigen in mammary epithelium under the control of the MMTV-LTR promoter develop multifocal metastatic mammary tumors with a reported average tumor latency $\left(\mathrm{T}_{50}\right)$ of 53 days (13). Female MMTV-PyV mT mice were treated twice weekly with Fc:T $\beta$ RII $(5 \mathrm{mg} / \mathrm{kg})$ or control IgG from 21 to 110 days of age. Mice treated with or without Fc:TßRII developed mammary tumors with a $\mathrm{T}_{50}$ of 56 days (Figure 1a). However, treatment with Fc:TßRII resulted in histologic alterations in the primary tumors. At 70 days, control glands displayed solid sheets of tumor cells. In contrast, Fc:T $\beta$ RII-treated mammary glands displayed cystic tumors containing secretions (Figure 1b). Mammary glands from wildtype FVB mice treated with Fc:T $\beta$ RII also displayed increased ductal secretions, a result reminiscent of the precocious alveolar differentiation exhibited by transgenic mice expressing a dominant negative TRRII in mammary epithelium $(23,24)$. At 110 days, mice treated with Fc:T $\beta R I I$ displayed tumors in all 10 mammary glands, as did IgG-treated mice. Total tumor weight for mice treated with Fc:T $\beta R I I$ was $16.3 \pm 3.2$ g, compared with $17.9 \pm 2.4 \mathrm{~g}$ for mice treated with $\mathrm{IgG}$ (unpaired Student $t$ test, $P=0.11$ ). Immunoblot analysis of 1:100 dilutions of mouse serum detected approximate Fc:T $\beta$ RII levels between 6.6 and $66 \mathrm{nM}$ (data not shown). 


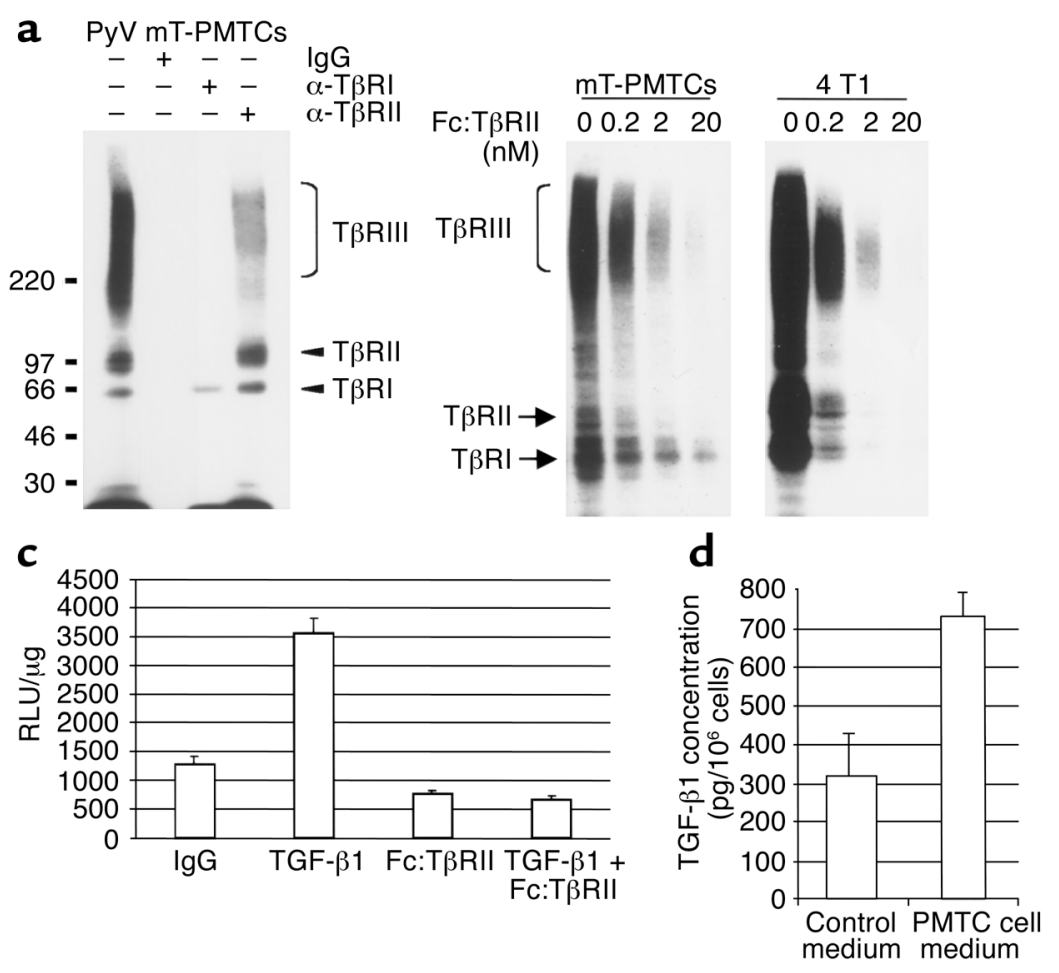

b
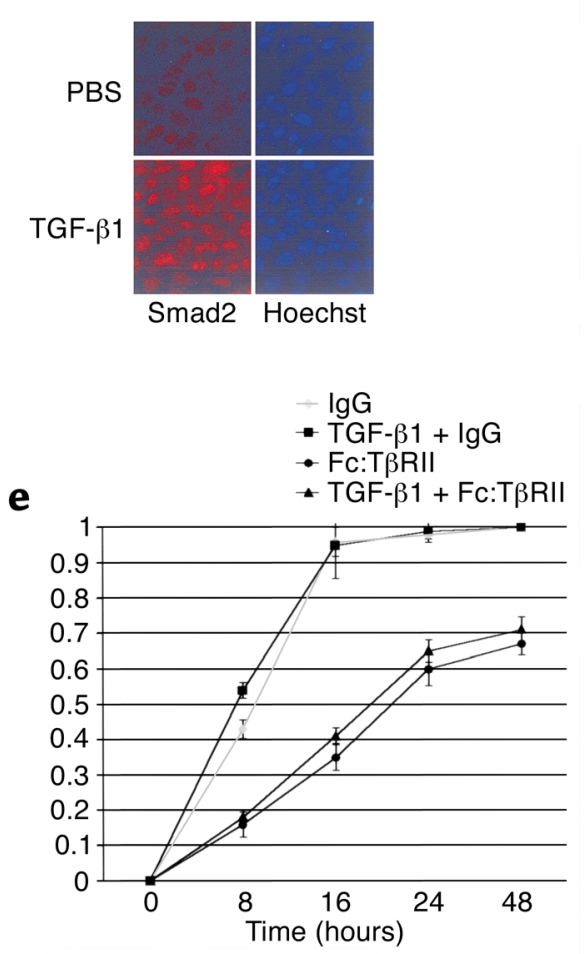

f

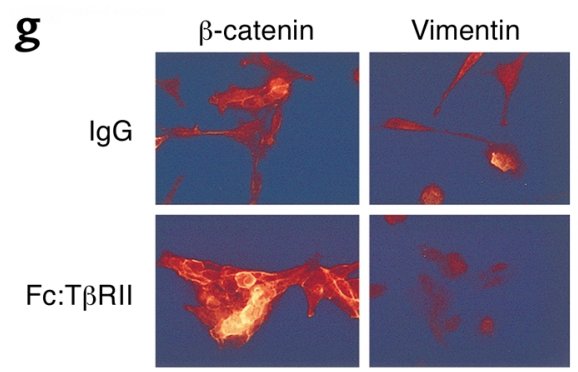

\section{Figure 2}

Fc:T $\beta$ RII inhibits autocrine TGF- $\beta$ signaling in PyV mT PMTCs. (a) Left panel: PMTCs were affinity labeled with ${ }^{125}$ I-TGF- $\beta 1$, resolved directly (lane 1) or immunoprecipitated with the indicated T $\beta R$ antibodies or IgG. Right panel: PMTCs and 4T1 cells were labeled with ${ }^{125}$-TGF $\beta 1$ in the presence of the indicated concentrations of Fc:T $\beta$ RII. (b) Immunofluorescent detection of Smad2 in PMTCs treated with PBS or TGF- $\beta 1$. (c) PMTCs transfected with a 3TP-Lux were treated with Fc:T $\beta R I I$, TGF- $\beta 1$, or both. The results are presented as relative light units $(\mathrm{RLUs}) / \mu \mathrm{g}$ of total protein. The values represent the average of 3 experiments performed in duplicate, \pm SE. (d) PMTCs or PMECs were cultured in serum-free medium for 24 hours. Conditioned medium was collected and analyzed for TGF- $\beta 1$ using ELISA. Results are shown as an average of 5 experiments, analyzed in triplicate. (e) PMTCs were grown to confluency and wounded. The results are presented as the percentage of the total area of the original wound enclosed by cells and represent the average \pm SD obtained from five experiments, analyzed in triplicate. (f) Transwell assays were performed using PMTCs, 4T1 cells, or EMT6 cells. The number of cells migrating to the lower side of the filter in controls was given the value of 1 , such that migration of cells is represented as a fraction of control. Values shown are the average $( \pm$ SE) of triplicate transwells in three experiments. $(\mathbf{g})$ Immunocytochemical detection of $\beta$-catenin or vimentin in PMTCs cultured in the presence of the indicated factors. Representative photographs are shown.

Increased apoptosis was observed in tumors from 10 -week-old mice treated with Fc:T $\beta$ RII $(2.4 \%)$ compared with controls $(0.9 \% ; P=0.024 ; n=6$ per group; unpaired Student $t$ test), as determined by TUNEL analysis (Figure 1c). However, treatment with Fc:T $\beta R I I$ did not alter the rate of tumor cell proliferation as assessed by BrdU incorporation $(P=0.08$; $n=6$ per group). These results were confirmed in purified PMTCs harvested from 110-day-old MMTV$\mathrm{PyV} \mathrm{mT}$ mice. BrdU incorporation in PMTCs in culture was not altered by TGF- $\beta 1(14.3 \% \pm 1.07 \%)$, by
Fc:T $\beta$ RII $(15.57 \% \pm 1.52 \%)$, or by a combination of both $(15.62 \% \pm 1.52 \%)$, as compared with PMTCs treated with $\operatorname{IgG}(15.1 \% \pm 1.15 \%)$.

Because mammary tumorigenesis induced by the $\mathrm{PyV}$ $\mathrm{mT}$ antigen has been shown to depend on the Shc, phosphatidylinositol-3 kinase (PI3K), and Src signaling pathways $(25,26)$, we investigated whether Fc:T $\beta R I I$ interfered with these mechanisms in the primary tumors. Tumor contents of Shc and p85, the regulatory subunit of PI3K, were unaffected by Fc:T $\beta$ RII (Figure 1d). Src expression, detected by immunoblot 
Table 1

Fc:T $\beta$ RII inhibits tumor cell extravasation and lung metastases

$\begin{array}{lcccc} & \text { WT } & & \text { MMTV-PyV } m T & \\ \text { Treatment: } & \text { Fc:T } \beta R I I & \text { Fc:T } \beta R I^{\mathrm{A}} & \operatorname{IgG}^{\mathrm{A}} & P \text { value } \\ \text { No. colonies } & 0 & 11.0 \pm 2.6 & 71.8 \pm 8.3 & 0.005 \\ \text { No. lung mets } & 0 & 2.9 \pm 1.5 & 28.6 \pm 4.1 & 0.004 \\ \text { Lung weight } & 0.32 \pm 0.02 \mathrm{~g} & 0.34 \pm 0.02 \mathrm{~g} & 0.42 \pm 0.02 \mathrm{~g} & 0.017 \\ \text { MMP (tissue) } & 2.16 \pm 0.81 & 23.06 \pm 2.89 & 29.46 \pm 4.37 & 0.024 \\ \text { MMP (PMTCs) } & \mathrm{N} / \mathrm{A} & 2.55 \pm 0.333 & 5.19 \pm 0.87 & 0.008\end{array}$

The number of colonies derived from circulating blood of 70-day-old wild-type or Py $V m T$ mice was quantified (see Figure $3 b$ ). Values shown are the average number of colonies derived per mouse, $n=6$ per condition. The average number of macroscopic surface lung metastases per mouse was determined and counted by two different individuals (R.S. Muraoka and E. Easterly) in a blinded fashion; $n>10$ per condition. Wet lung weight was determined at time of death; $n>10$ per condition. Relative combined activity of MMP- 2 and MMP-9 in mammary tissue extracts or cell lysates of purified PMTCs was determined using ELISA-based detection of MMP cleavage products (see Methods). Values represent the relative MMP activity in lysates compared with $0.2 \mu \mathrm{g}$ of purified recombinant MMP-2. Values shown are the average of three independent experiments. $n=12$ per condition (MMP: tissue); $n=8$ per condition (MMP: PMTCs). AStatistical analysis performed using unpaired Student $t$ test, comparing figures for Fc:T $\beta R$ II and IgG. WT, wild-type; mets; metastases.

analysis, and in vitro kinase activity of Src were also unaffected (data not shown). Phosphorylation of Akt, a serine/threonine kinase whose activity is induced by

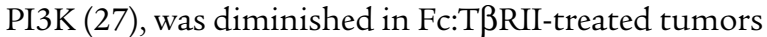
approximately 2.4-fold. Phosphorylation of the Akt target FKHRL1, a Forkhead transcription factor that induces genes involved in cell death $(28,29)$, was also reduced 5.2-fold in Fc:T $\beta$ RII-treated tumors. Consistent with previous reports showing that inhibition of Akt results in loss of FKHRL1 phosphorylation and translocation to the nucleus $(28,30)$, treatment with Fc:T $\beta R I I$ induced nuclear localization of FKHRL1 in PMTCs (Figure 1e).

PyV mT mammary tumor cells exhibit evidence of autocrine TGF- $\beta$ signaling. Although proliferation of tumor cells was unaffected by Fc:T $\beta$ RII, this was not due to lack of functional TGF- $\beta$ receptors. PMTCs were affinitylabeled with ${ }^{125}$ I-TGF- $\beta 1$, resulting in three species with the characteristic mobility and molecular weight of type I, II, and III TGF- $\beta$ receptors (Figure $2 a$ ). TGF- $\beta$ binding was competed with nanomolar concentrations of Fc:T $\beta$ RII. 4T1 mouse mammary tumor cells also expressed TGF- $\beta$ receptors, whose interactions with labeled ligand were inhibited by Fc:T $\beta$ RII. In PMTCs, exogenous TGF- $\beta$ induced nuclear localization of Smad2 (Figure $2 b$ ), as well as transcription from the TGF- $\beta$-responsive reporter 3 TP-Lux (Figure $2 c$ ). Both basal and TGF- $\beta$-induced reporter activity were blocked by Fc:T $\beta$ RII (Figure $2 c)$. These results suggest that TGF- $\beta$ receptor-mediated signaling is intact in $P y V m T$ mouse-derived cells. In addition, PMTCs synthesize and secrete close to $1 \mathrm{ng} / \mathrm{ml} / 24 \mathrm{~h}$ of TGF- $\beta 1$ (Figure 2d). Fc:T $\beta$ RII impaired ligand-induced and basal motility of PMTCs (Figure 2e). The rate of wound closure was the same in the presence or absence of mitomycin C, suggesting that these effects were independent of cell proliferation (not shown). In transwell invasion assays, PMTCs, 4T1, and EMT6 BALB/c mammary tumor cells migrated through extracellular matrix (Matrigel) to the opposite sides of filters. In each case, tumor cell migration was impaired by Fc:T $\beta$ RII (Figure 2f). Cultured PMTCs treated with TGF- $\beta 1$ and Fc:T $\beta$ RII or with TGF- $\beta 1$ alone were analyzed for expression of either $\beta$-catenin or vimentin using immunocytochemistry (Figure $2 \mathrm{~g}$ ). PMTCs treated with TGF- $\beta 1$ and Fc:T $\beta$ RII together displayed abundant $\beta$-catenin expression that was localized to the cell membranes. However, $\beta$-catenin expression was downregulated in PMTCs treated with TGF- $\beta 1$. Vimentin expression, a marker of epithelial-to-mesenchymal transition, was observed in TGF- $\beta 1$-treated PMTCs, but was not observed in PMTCs treated with Fc:T $\beta R I I$ and TGF- $\beta$ together.

Blockade of TGF- $\beta$ with Fc:T $\beta R I I$ reduces mammary tumor cell intravasation and lung metastases. At 110 days, lungs from transgenic mice were collected and examined for surface metastases. Lung surface metastases were observed in control mice and mice treated with Fc:T $\beta$ RII. However, Fc:T $\beta$ RII-treated mice displayed significantly lower wet lung weight (Table 1) and tenfold fewer lung surface metastases than controls (Figure 3a). All lung metastases expressed the MMTV-PyV $\mathrm{mT}$ transgene product as measured by immunohistochemistry (Figure 3a). Tumor cell intravasation was measured by collecting blood via atrial puncture and culturing the serum and buffy-coat layers (20). After 1 week, we determined the number of epithelial colonies (Figure $3 \mathrm{~b})$. Blood from wild-type mice produced no colonies. However, blood from control and Fc:T $\beta$ RII-treated mice grew an average of $71.3 \pm 8.3$ and $11.0 \pm 2.6$ colonies per mouse, respectively $(n=6$ per group; $P<0.009$; unpaired student $t$ test). Although we cannot rule out the possibility that Fc:T $\beta R I I$ affected the survival of circulating tumor cells, these results suggest that Fc:T $\beta$ RII might interfere with the ability of tumor cells to migrate from the primary tumor and intravasate. Since TGF- $\beta$ can also induce the expression of MMPs (10), we examined MMP activity in mammary tumor extracts. When normalized for protein content, the relative combined MMP-2 and MMP-9 activities were reduced in lysates prepared

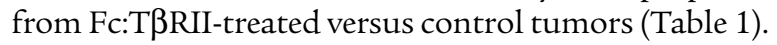
The relative levels of MMP activity were measured in extracts prepared from purified PMTCs treated in culture with Fc:T $\beta$ RII or with control IgG. We found decreased MMP activity in Fc:T $\beta$ RII-treated PMTC lysates compared with IgG-treated PMTC lysates, consistent with the hypothesis that Fc:T $\beta$ RII may impair MMP activity in tumor cells.

To confirm that the antimetastatic effect of Fc:T $\beta$ RII was not limited to $P y V m T$ mice, we studied the effect of Fc:TßRII on 4T1 and EMT6 mammary tumor cell metastases. These transplantable mouse 
a

$\lg G$
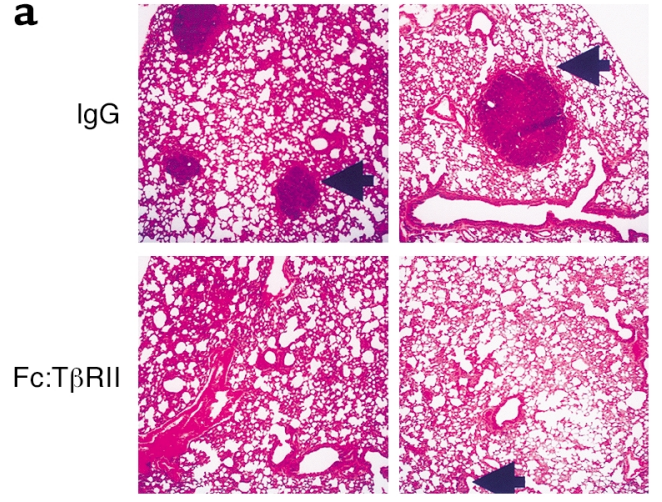

b

WT

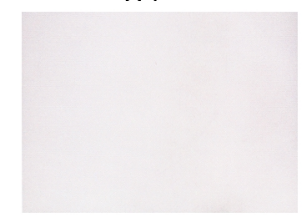

Fc:T $\beta R I I$
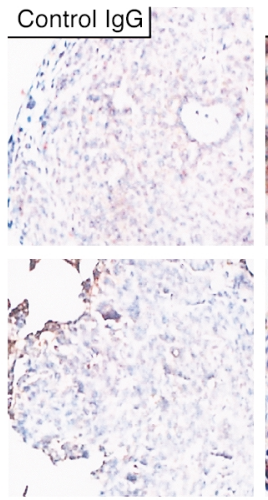

PyV mT

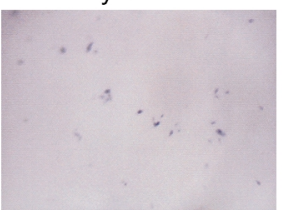

Fc:T $\beta R I I$
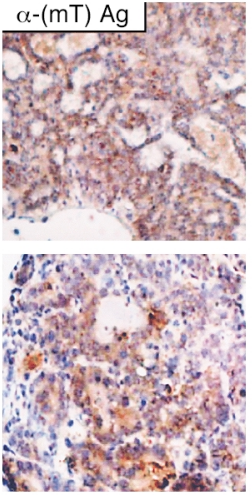

PyV mT

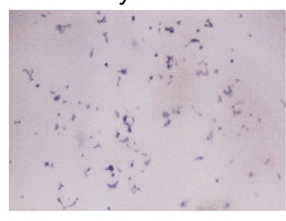

$\lg G$

\section{Figure 3}

Fc:T $\beta$ RII decreases PyV $m T$ mouse tumor cell intravasation and lung metastases. (a) Hematoxylin and eosin-stained lung sections (left panels) or $\mathrm{mT} \mathrm{Ag} \mathrm{immunohistochemical}$ analysis (right panels) of lungs harvested from 110-day-old PyV mT mice. Arrows point to lung metastases. Immunohistochemistry was performed using normal mouse IgG or pAb 762 against middle $T$ antigen $[\alpha-(m T) A g]$. (b) Photomicrographs of hematoxylinstained tumor cell colonies harvested from the blood of 110-day-old wildtype or PyV $m T$ mice treated with normal mouse IgG or Fc:T $\beta R I I$. tumor cells express high levels of TGF- $\beta$ ligands and receptors and exhibit enhanced motility in response to exogenous TGF- $\beta$ (19). Furthermore, expression of a dominant negative truncated T $\beta$ RII in $4 \mathrm{~T} 1$ cells markedly restricted lung metastases (31). 4T1 or EMT6 cells $\left(0.5 \times 10^{5}\right)$ were implanted into the mammary glands of virgin 6-week-old BALB/c mice. Mice were treated twice weekly with Fc:T $\beta$ RII, and primary tumors were resected after 10 days. Fc:T $\beta R I$ was continued for 8 additional weeks. After only three doses of Fc:T $\beta R I I, 4 T 1$ and EMT6 tumors were smaller than controls (Figure 4a), suggesting that tumor growth in vivo is retarded by TGF- $\beta$ blockade. Although this result is in contrast with the results obtained using Fc:T $\beta R I I$ in the MMTV-PyV mT mice, the difference may be due to differential effects of Fc:T $\beta$ RII on an experimental tumor derived from metastatic tumors (i.e., 4T1 and EMT6) compared with a tumor that has evolved de novo from preneoplastic mammary epithelial cells (i.e., MMTV-PyV mT mammary glands). We found BrdU incorporation was not significantly different in 4T1 tumors treated with Fc:T $\beta R I I(17.9 \% \pm 2.4 \%)$ compared with IgG-treated $4 \mathrm{~T} 1$ tumors $(18.4 \% \pm 2.2 \% ; P=0.14$; unpaired student $t$ test), suggesting that tumor cell proliferation was not affected by Fc:T $\beta$ RII. Mice treated with Fc:TßRII had 3.1-fold $(P<0.006)$ and 2.6-fold $(P<0.002$; unpaired Student $t$ test $)$ fewer $4 \mathrm{~T} 1$ and EMT6 lung metastases, respectively, compared with IgG-treated mice (Figure $4 b$ ). In addition to the lungs, 4T1 cells have been reported to metastasize to other sites in $\mathrm{BALB} / \mathrm{c}$ mice, including the liver. Mice treated

\section{Figure 4}

Fc:T $\beta$ RII inhibits 4 T1 and EMT6 primary tumor size and lung metastases. Tumor cells $\left(0.5 \times 10^{5} 4\right.$ T 1 or EMT6 $)$ were injected into the mammary fat pad of BALB/c mice. Mice were treated twice weekly with $\mathrm{Fc}: T \beta R I I$. (a) After 10 days, primary tumors were resected. Arrows indicate the lymph nodes of the number 4 mammary gland. The average tumor volume ( $n=8$ per condition), calculated by the formula vol-ume $=$ width $^{2} \times$ length/2, is indicated in the lower right corner. ${ }^{*} P=0.011 ;{ }^{*} P=0.05$. (b) Lungs were collected at 8 weeks and examined for lung surface metastases. Arrows indicate metastases. The average number of metastases per mouse is shown in the bottom right corner of each panel $(n=8$ per condition). ${ }^{*} P=0.026 ;{ }^{*} P=0.034$. a

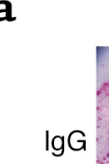

b
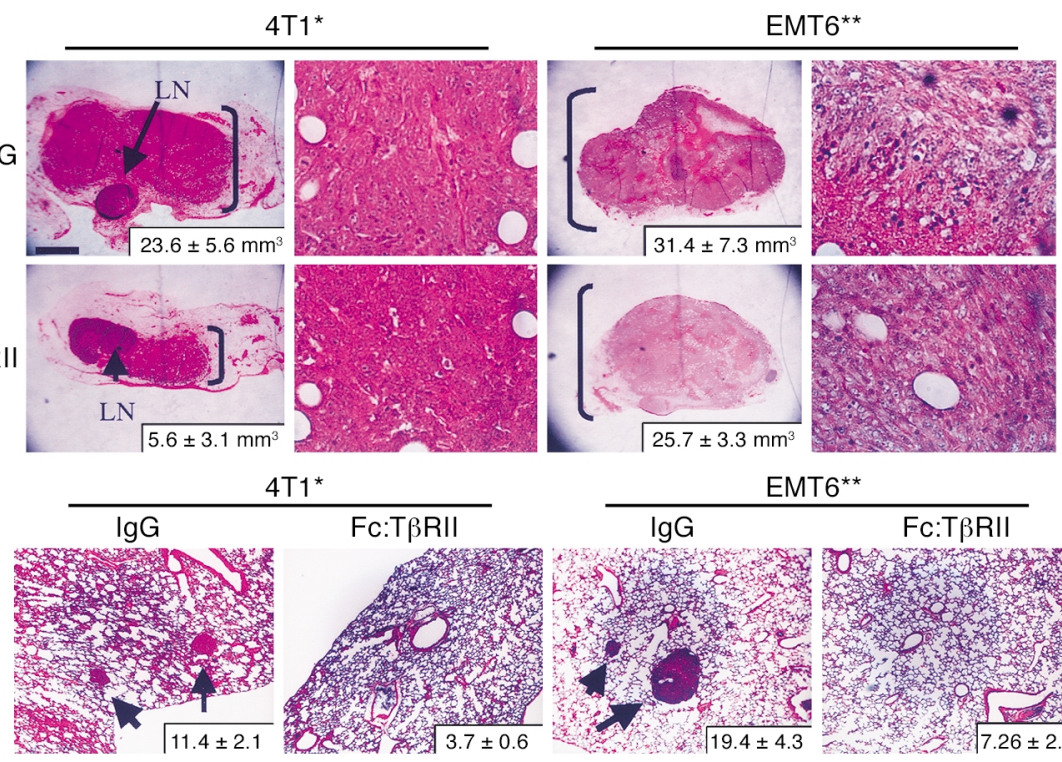

EMT6** $^{\star 2}$

$\lg G \quad F c: T \beta R I I$

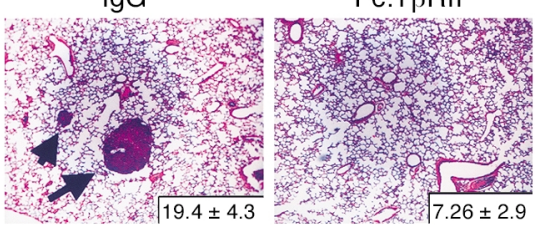


a

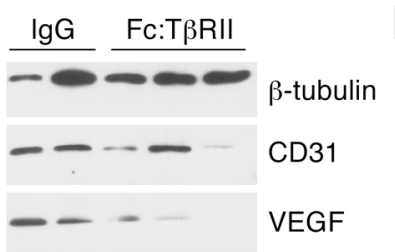

c Bright field $\times 2$

$\lg G$
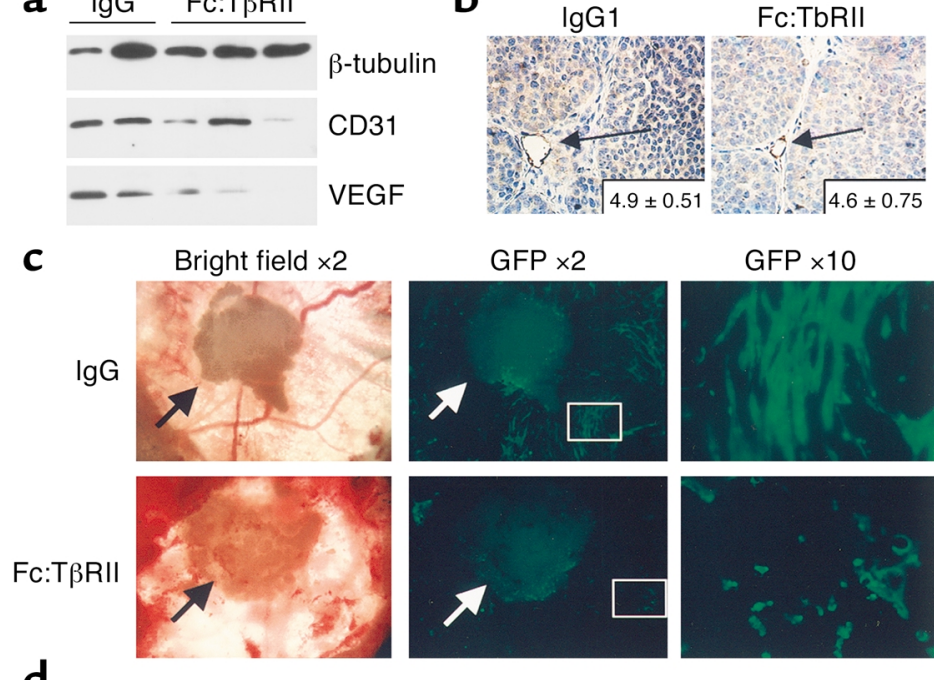

d

$\lg G$

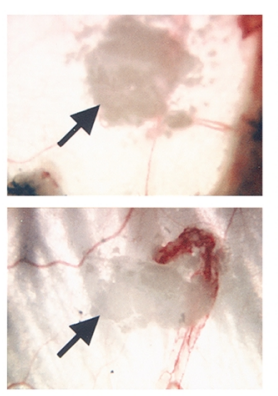

e

Bright field $\times 2$

$\lg G$
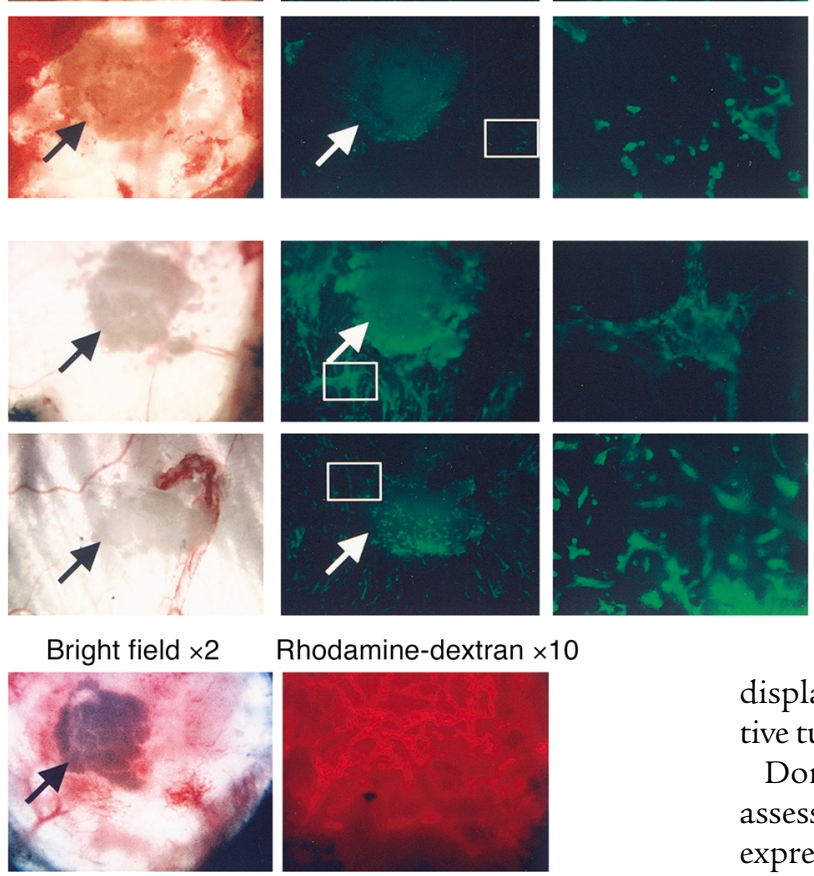

\section{Figure 5}

Fc:T $\beta$ RII alters tumor cell density and invasiveness but not tumor angiogenesis. (a) Tumor lysates from PyV mT mice were subjected to Western blot analysis using Ab's against $\beta$-tubulin, CD31, or VEGF. The VEGF isoform shown is mouse VEGF-A 120 , migrating at $14 \mathrm{kDa}$. (b) Immunohistochemical detection of CD31 in tumors harvested from 110day-old MMTV/PyV $m T$ mice treated with normal mouse IgG or Fc:T $\beta$ RII. The average number of vessels per $\times 400$ field is indicated in the lower-right corner of each panel. ( $n=10$ random tumor fields from three mice per condition.) Dorsal skin window analysis of in vivo tumor angiogenesis. One hundred 4T1-GFP cells were implanted into dorsal window chambers in BALB/c mice along with Fc:T $\beta$ RII-, IgG-, or PBSreleasing pellets on day 0 . Arrows indicate location of the pellet. On day 15, mice were administered rhodamine-conjugated dextran. Digital photomicrographs were taken on days 5 (c), 10 (d), and 15 (e) under green field (to visualize 4T1-GFP tumor cells), red field (to visualize the rhodaminelabeled vasculature), and bright field. Relative tumor density was calculated using the following equation: (average number of fluorescent pixels per sample in $\mathrm{Fc}_{\mathrm{C}} \mathrm{T} \beta \mathrm{R}$ II group)/ (average number of fluorescent pixels per sample in IgG control group and PBS control group combined). The relative vascular density was determined using the following equation: (number of fluorescent pixels in test sample)/(number

of fluorescent pixels in IgG and PBS controls).
Rhodamine-dextran $\times 10$

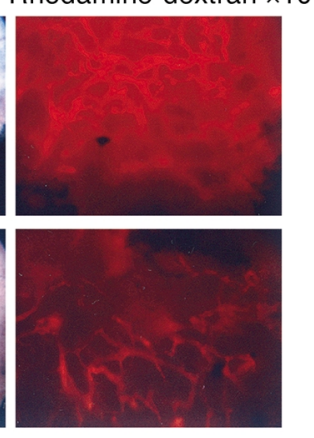

with Fc:T $\beta$ RII displayed fewer surface liver metastases $(4.4 \pm 1.2)$ compared with IgG-treated mice (6.4 \pm 1.31 ; $P<0.04$. unpaired Student $t$ test, $n=5$ ).

Antimetastatic effect of soluble Fc:T $\beta R I I$ is not associated with inhibition of tumor angiogenesis. TGF- $\beta$ may promote angiogenesis via multiple mechanisms, including increased production of VEGF and recruitment of perivascular pericytes, among others (32). Western analysis of primary tumor extracts from control or Fc:T $\beta$ RII-treated MMTV-PyV mT mice demonstrated the presence of VEGF-A isoforms at 42, 27, and $14 \mathrm{kDa}$ (Figure 5a and data not shown). Expression of CD31, a marker for endothelial cells, was observed in tumors treated with or without Fc:T $\beta$ RII. Quantification by immunohistochemistry of the number of CD31-positive vessels in situ was similar in tumors treated or not treated with Fc:T $\beta$ RII $(P=0.19, n=5$; Figure $5 \mathrm{~b})$. Similarly, Fc:T $\beta$ RII-treated 4T1 and EMT6 tumors

displayed no differences in the number of CD31-positive tumor vessels compared with controls (not shown).

Dorsal skin window assays (21) were performed to assess early stages of angiogenesis. 4T1 tumor cells expressing GFP (22) were implanted into a dorsal window chamber. A hydron pellet impregnated with Fc:TßRII, PBS, or normal mouse IgG was implanted in the chamber. 4T1 tumor formation and vascularization were monitored over a 15-day period. At day 5, the 4T1GFP cells in the window chamber containing a control hydron pellet were present at a higher density than those in the presence of an Fc:T $\beta$ RII pellet. Upon higher magnification, Fc:T $\beta$ RII-treated cells were rounded compared with the spindle-shaped 4T1-GFP cells in control chambers. At day 10, Fc:T $\beta$ RII-treated cells remained as rounded, single cells (Figure $5 \mathrm{c}$ ). At day 14, mice were administered rhodamine-dextran intravenously to visualize vascularization of tumors. There were no differences in vascularity of control versus Fc:T $\beta$ RII-treated 4T1-GFP tumors as measured by rhodamine-fluorescent pixels. However, tumor density determined by the number of GFP-fluorescent pixels was decreased in the Fc:T $\beta$ RII-treated mice (Figure $5 \mathrm{~d}$ and Table 2), suggesting that Fc:T $\beta$ RII may have an effect on tumor cells independent of any detectable effect on endothelial cell recruitment and/or new vessel formation.

\section{Discussion}

Blockade of TGF- $\beta$ signaling with soluble Fc:T $\beta$ RII inhibited the formation of distant metastases in three 
Table 2

Vascular density is not reduced by Fc:T $\beta R I I$

\begin{tabular}{|c|c|c|c|c|c|c|}
\hline & \multicolumn{2}{|c|}{ Day 5} & \multicolumn{2}{|c|}{ Day 10} & \multicolumn{2}{|c|}{ Day 15} \\
\hline & $\operatorname{lgG}$ & Fc:T $\beta$ RII & $\lg G$ & Fc:TRRII & $\lg G$ & Fc:T $\beta$ RII \\
\hline Tumor density & $1,742 \pm 141$ & $597 \pm 96$ & $4,796 \pm 274$ & $865 \pm 174$ & $22,988 \pm 1,412$ & $14,765 \pm 1,633$ \\
\hline Vascular density & N/A & $\mathrm{N} / \mathrm{A}$ & N/A & $\mathrm{N} / \mathrm{A}$ & $27,861 \pm 1,955$ & $26,433 \pm 2,720$ \\
\hline
\end{tabular}

The density of 4T1 tumor cells in dorsal skin window chambers was quantified at the indicated time points as the average number of GFP-generated fluorescent pixels per $\times 20$ field (see Figure 5, c and d) using Scion Image software, $n=6$ per condition. The vascular density of 4 T1 tumors in dorsal skin window chambers was quantified as the average number of rhodamine-generated fluorescent pixels per $\times 20$ field (see Figure $5 \mathrm{~d}$ ) using Scion Image software, $n=6$ per condition.

experimental models of breast cancer. Although responsive to exogenous TGF- $\beta$ (Figure 2 ), inhibition of TGF- $\beta$ signaling using Fc:T $\beta$ RII did not alter cellular proliferation in the tumor cells used in this study, neither in vitro nor in vivo (Figure 1c), suggesting that the antimetastatic effects of Fc:T $\beta R I I$ in vivo were independent of tumor cell proliferation. However, treatment with Fc:TßRII inhibited tumor cell motility (Figure 2) and intravasation (Figure 3), inhibited MMP activity in tumors (Table 1), and increased cancer cell apoptosis in situ (Figure 1). These data suggest that TGF- $\beta$ signaling contributes to metastasis (33) and that Fc:TßRII (or other mechanisms of systemic inhibition of TGF- $\beta 1$ signaling) may be an effective treatment for the prevention of tumor cell metastasis. These data are consistent with studies in which inactivating mutations in the TRRII gene in colon cancers correlate with a low invasive potential; introduction of exogenous TRRII into these colon cancer cells increased tumor cell invasion (9). Approximately $90 \%$ of colon cancers with microsatellite instability have inactivating mutations of T $\beta$ RII (34), which correlates with longer patient survival (35), implying that loss of TGF- $\beta$ signaling may limit systemic metastases.

Mammary tumors from mice treated with Fc:TßRII displayed a higher rate of apoptosis than that observed for control-treated tumors. Interestingly, we found that Fc:T $\beta$ RII inhibited phosphorylation of Akt on serine 473. Phosphorylation on serine 473 is required for maximal Akt kinase activity resulting in subsequent phosphorylation of multiple downstream targets of Akt, such as p21, p27, GSK3 $\beta$, IKK $\alpha$, and FKHRL1. Phosphorylation of FKHRL1 by Akt results in the cytoplasmic retention (i.e., nuclear exclusion) of FKHRL1, thus preventing FKHRL1-induced transcription of death-associated genes. Therefore, inhibition of Akt might conceivably result in enhanced nuclear localization of FKHRL1 and increased cell death. The data reported herein suggest that, by inhibiting the phosphorylation/activation of Akt, Fc:T $\beta$ RII treatment resulted in increased nuclear localization of FKHRL1. This correlated with an increase in the number of apoptotic nuclei in situ in tumors treated with Fc:T $\beta$ RII. These data are consistent with reports published previously demonstrating the reported induction of PI3K by TGF- $\beta$ ( 42 ). TGF- $\beta$-neutralizing Ab's are known to inhibit phosphorylation of Akt at serine 473 in 4T1 and EMT6 cells (19). More recently, it was shown that TGF- $\beta$ enhances epithelial cell survival via Akt-induced phosphorylation and nuclear exclusion of FKHRL1 (30). Therefore, inhibition of TGF- $\beta$-induced AKT activity by Fc:T $\beta$ RII may enhance tumor cell death and limit metastatic progression.

The data presented cannot rule out the possibility that Fc:T $\beta$ RII-mediated blockade of TGF- $\beta$ signaling in immune effector cells might lead to eradication of tumor, as demonstrated recently in mice expressing dominant negative TRRII in $\mathrm{CD}^{+}$and $\mathrm{CD}^{+} \mathrm{T}$ cells (36). It is difficult to compare the results presented herein with the powerful results reported by Gorelick and Flavell (36), who used a highly aggressive, poorly immunogenic, transplantable model of melanoma, while our studies examined the endogenous formation of breast tumors from preneoplastic mammary epithelium. This difference may present differences in the mechanisms by which the immune system recognize tumor cells. Additionally, TGF- $\beta$ may have different effects on tumor progression at different stages of tumorigenesis. TGF- $\beta$ is thought to act as a tumor suppressor early in tumorigenesis, perhaps by inhibiting cell proliferation. Later in tumor progression, however, TGF- $\beta$ may not inhibit tumor cell proliferation and may actually enhance tumor cell invasion and the tumor microenvironment, while inhibiting tumoricidal activity of the immune system, thus enhancing tumor progression. It is conceivable that Fc:T $\beta$ RII would interfere with each of these effects of TGF- $\beta$ on tumor progression. Nonetheless, the studies showing inhibition of TGF- $\beta$ signaling in T cells, taken together with data presented herein, demonstrate a beneficial effect in tumor metastasis prevention by inhibition of TGF- $\beta$ signaling.

Our studies suggest that inhibition of TGF- $\beta$ signaling using Fc:T $\beta R I I$ may not interfere with tumor angiogenesis in vivo. This finding is in contrast to other studies using TGF- $\beta$ inhibitors $(10,32)$. TGF- $\beta$ is predominantly involved in smooth muscle cell differentiation and migration leading to pericyte recruitment and vessel stabilization (37). However, the majority of intratumor neovessels lack periendothelial smooth muscle cells (38), potentially explaining our inability to detect a reduction of in situ vascular density in endogenously arising tumors and in dorsal skin window chambers. 
In summary, the mechanisms by which TGF- $\beta$ can promote late stages of tumor progression represent testable molecular targets for novel interventions like Fc:T $\beta$ RII. Our results suggest that inhibition of TGF- $\beta$ signaling results in decreased metastasis of mammary tumors by impairing invasion, migration, and cellular survival. The lack of any obvious toxicity in mice treated with Fc:T $\beta R I I$ for 12 weeks suggests that Fc:T $\beta R I I$ or other inhibitors of the TGF- $\beta$ signaling pathway may prove to be powerful antimetastatic therapies.

\section{Acknowledgments}

The authors are grateful to Steven Dilworth for providing key reagents and Jean Simpson for histological analyses. This work was supported by NIH training grant T32 CA-09592 (R.S. Muraoka), U.S. Army DAMD 17-98-1-8263 Pre-doctoral Training Award (N. Dumont), NIH grant R01 CA-62212 (C.L. Arteaga), and Vanderbilt-Ingram Comprehensive Cancer Center support grant CA68485.

1. Hanahan, D., and Weinberg, R.A. 2000. The hallmarks of cancer. Cell. 100:57-70.

2. Massague, J. 1998. TGF-beta signal transduction. Annu. Rev. Biochem. 67:753-791.

3. Derynck, R., Akhurst, R.J., and Balmain, A. 2001. TGF-beta signaling in tumor suppression and cancer progression. Nat. Genet. 29:117-129.

4. Pierce, D.F., Jr., et al. 1993. Inhibition of mammary duct development but not alveolar outgrowth during pregnancy in transgenic mice expressing active TGF-beta 1. Genes Dev. 7:2308-2317.

5. Jhappan, C., et al. 1993. Targeting expression of a transforming growth factor beta 1 transgene to the pregnant mammary gland inhibits alveolar development and lactation. EMBOJ. 12:1835-1845.

6. Pierce, D.F., Jr., et al. 1995. Mammary tumor suppression by transforming growth factor beta 1 transgene expression. Proc. Natl. Acad. Sci. USA. 92:4254-4258

7. Cui, W., et al. 1996. TGFbeta1 inhibits the formation of benign skin tumors, but enhances progression to invasive spindle carcinomas in transgenic mice. Cell. 86:531-542.

8. Oft, M., et al. 1996. TGF-beta1 and Ha-Ras collaborate in modulating the phenotypic plasticity and invasiveness of epithelial tumor cells. Genes Dev. 10:2462-2477.

9. Oft, M., Heider, K.H., and Beug, H. 1998. TGFbeta signaling is necessary for carcinoma cell invasiveness and metastasis. Curr. Biol. 8:1243-1252.

10. Dumont, N., and Arteaga, C.L. 2000. Transforming growth factor-beta and breast cancer: tumor promoting effects of transforming growth factor-beta. Breast Cancer Res. 2:125-132.

11. Cosgrove, D., et al. 2000. Integrin alpha1beta1 and transforming growth factor-beta 1 play distinct roles in alport glomerular pathogenesis and serve as dual targets for metabolic therapy. Am.J. Pathol. 157:1649-1659.

12. George, J., Roulot, D., Koteliansky, V.E., and Bissell, D.M. 1999. In vivo inhibition of rat stellate cell activation by soluble transforming growth factor beta type II receptor: a potential new therapy for hepatic fibrosis. Proc. Natl. Acad. Sci. USA. 96:12719-12724.

13. Guy, C.T., Cardiff, R.D., and Muller, W.J. 1992. Induction of mammary tumors by expression of Polyomavirus middle T oncogene: a transgenic mouse model for metastatic disease. Mol. Cell Biol. 12:954-961.

14. Brantley, D.M., et al. 2000. Dynamic expression and activity of NF-kappaB during post-natal mammary gland morphogenesis. Mech. Dev. 97:149-155.

15. Dilworth, S.M., and Horner, V.P. 1993. Novel monoclonal antibodies that differentiate between the binding of pp60c-src or protein phosphatase 2A by Polyomavirus middle T antigen. J. Virol. 67:2235-2244.
16. Muraoka, R.S., et al. 2001. Cyclin-dependent kinase inhibitor p27(Kip1) is required for mouse mammary gland morphogenesis and function. J. Cell Biol. 153:917-932.

17. Lenferink, A.E., Busse, D., Flanagan, W.M., Yakes, F.M., and Arteaga, C.L. 2001. ErbB2/neu kinase modulates cellular p27(Kip1) and cyclin D1 through multiple signaling pathways. Cancer Res. 61:6583-6591.

18. Dumont, N., O'Connor-McCourt, M.D., and Philip, A. 1995. Transforming growth factor-beta receptors on human endometrial cells: identification of the type I, II, and III receptors and glycosyl-phosphatidylinositol anchored TGF-beta binding proteins. Mol. Cell Endocrinol. 111:57-66.

19. Bakin, A.V., Tomlinson, A.K., Bhowmick, N.A., Moses, H.L., and Arteaga, C.L. 2000. Phosphatidylinositol 3-kinase function is required for transforming growth factor beta-mediated epithelial to mesenchymal transition and cell migration. J. Biol. Chem. 275:36803-36810.

20. Wyckoff, J.B., Jones, J.G., Condeelis, J.S., and Segall, J.E. 2000. A critical step in metastasis: in vivo analysis of intravasation at the primary tumor. Cancer Res. 60:2504-2511.

21. Huang, Q., et al. 1999. Noninvasive visualization of tumors in rodent dorsal skin window chambers. Nat. Biotechnol. 17:1033-1035.

22. Lin, P., et al. 1997. Inhibition of tumor angiogenesis using a soluble receptor establishes a role for Tie2 in pathologic vascular growth. J. Clin. Invest. 100:2072-2078.

23. Bottinger, E.P., Jakubczak, J.L., Haines, D.C., Bagnall, K., and Wakefield, L.M. 1997. Transgenic mice overexpressing a dominant-negative mutant type II transforming growth factor beta receptor show enhanced tumorigenesis in the mammary gland and lung in response to the carcinogen 7,12-dimethylbenz-[a]-anthracene. Cancer Res. 57:5564-5570.

24. Gorska, A.E., Joseph, H., Derynck, R., Moses, H.L., and Serra, R. 1998. Dominant-negative interference of the transforming growth factor beta type II receptor in mammary gland epithelium results in alveolar hyperplasia and differentiation in virgin mice. Cell Growth Differ. 9:229-238.

25. Guy, C.T., Muthuswamy, S.K., Cardiff, R.D., Soriano, P., and Muller, W.J. 1994. Activation of the c-Src tyrosine kinase is required for the induction of mammary tumors in transgenic mice. Genes Dev. 8:23-32.

26. Webster, M.A., et al. 1998. Requirement for both Shc and phosphatidylinositol 3' kinase signaling pathways in Polyomavirus middle T-mediated mammary tumorigenesis. Mol. Cell Biol. 18:2344-2359.

27. Datta, S.R., Brunet, A., and Greenberg, M.E. 1999. Cellular survival: a play in three Akts. Genes Dev. 13: 2905-2927.

28. Brunet, A., et al. 1999. Akt promotes cell survival by phosphorylating and inhibiting a Forkhead transcription factor. Cell. 96:857-868.

29. Nakamura, N., et al. 2000. Forkhead transcription factors are critical effectors of cell death and cell cycle arrest downstream of PTEN. Mol. Cell Biol. 20:8969-8982.

30. Shin, I., Bakin, A.V., Rodeck, U., Brunet, A., and Arteaga, C.L. 2001. Transforming growth factor beta enhances epithelial cell survival via Akt-dependent regulation of FKHRL1. Mol. Biol. Cell. 12:3328-3339.

31. McEarchern, J.A., et al. 2001. Invasion and metastasis of a mammary tumor involves TGF-beta signaling. Int. J. Cancer. 91:76-82.

32. Pepper, M.S. 1997. Transforming growth factor-beta: vasculogenesis, angiogenesis, and vessel wall integrity. Cytokine Growth Factor Rev. 8:21-43.

33. Miettinen, P.J., Ebner, R., Lopez, A.R., and Derynck, R. 1994. TGF-beta induced transdifferentiation of mammary epithelial cells to mesenchymal cells: involvement of type I receptors. J. Cell Biol. 127:2021-2036.

34. Markowitz, S., et al. 1995. Inactivation of the type II TGF-beta receptor in colon cancer cells with microsatellite instability. Science. 268:1336-1338.

35. Thibodeau, S.N., Bren, G., and Schaid, D. 1993. Microsatellite instability in cancer of the proximal colon. Science. 260:816-819.

36. Gorelik, L., and Flavell, R.A. 2001. Immune-mediated eradication of tumors through the blockade of transforming growth factor-beta signaling in T cells. Nat. Med. 7:1118-1122.

37. Hirschi, K.K., Rohovsky, S.A., and D’Amore, P.A. 1998. PDGF, TGF-beta, and heterotypic cell-cell interactions mediate endothelial cell-induced recruitment of $10 \mathrm{~T} 1 / 2$ cells and their differentiation to a smooth muscle fate. J. Cell Biol. 141:805-814.

38. Benjamin, L.E., Golijanin, D., Itin, A., Pode, D., and Keshet, E. 1999. Selective ablation of immature blood vessels in established human tumors follows vascular endothelial growth factor withdrawal. J. Clin. Invest. 103:159-165. 